\title{
Curved incision under the costal margin in retroperitoneal tumor surgery
}

\author{
YANG QIN, YONG YANG, HONG YANG and JUN LI \\ Department of Urinary Surgery, The Third Affiliated Hospital of Kunming Medical University, \\ Kunming, Yunnan 650118, P.R. China
}

Received February 2, 2015; Accepted March 24, 2015

DOI: $10.3892 / \mathrm{mco} .2015 .541$

\begin{abstract}
This study was conducted to investigate the value of using a curved incision under the costal margin in retroperitoneal tumor surgery. Data on 68 patients with retroperitoneal tumors were reviewed. Of the 68 patients, 32 underwent tumor removal via a curved incision under the costal margin (group A) and the remaining 36 patients underwent a mid-abdominal oblique incision at the waist (group B). The patient outcomes were compared between the two groups to analyze the effects of the different types of incision on the completeness of resection, duration of operation, intraoperative blood loss, postoperative recovery time and surgical complications. The tumors from the 32 patients in group A were all successfully removed and the outcomes of the patients in group A, regarding tumor removal, duration of operation, amount of blood lost and surgical complications, were superior to those of the patients in group B. A route of incision is required that adequately exposes the internal limit or lower boundary of the tumor and ensures adequate surgical field visualization. The 32 patients underwent retroperitoneal tumor removal via a curved incision under the costal margin, plus a longitudinal incision along the lower boundary of the tumor at the midline of the abdomen. All the patients underwent complete tumor resection and recovered well after surgery, without necrosis at the incision site. In terms of duration of the operation, blood transfusion volume, mean length of hospital stay, weight of tumor removed, perioperative mortality, postoperative intestinal fistulas and rate of complete surgical resection, the outcomes of the patients in group A were superior to those of group B.
\end{abstract}

\section{Introduction}

Retroperitoneal tumors are located in the retroperitoneal space. More specifically, 'primary' retroperitoneal tumors

Correspondence to: Dr Yang Qin, Department of Urinary Surgery, The Third Affiliated Hospital of Kunming Medical University, 519 Kunzhou Road, Kunming, Yunnan 650118, P.R. China

E-mail: qinyang22@qq.com

Key words: retroperitoneal tumor treatment, surgical exposure, curved incision under the costal margin refers to tumors that do not include those located in the pancreas, kidney, adrenal gland, or other substantive organs in the retroperitoneal space (1-3). Histologically, retroperitoneal tumors are classified into soft tissue, hematopoietic/lymphoid, urinary tract and germ cell tumors. Due to the location of retroperitoneal tumors, early diagnosis may be difficult. Even tumors that develop on the kidney, adrenal gland and other organs may grow slowly and asymptomatically and patients only receive treatment when the tumor grows to a certain size, appears in surrounding tissues and organs, or upon development of compression or obstruction of neighboring structures. By this time, the tumors are usually large, expansive and grow invasively, often with unclear boundaries between the tumor and adjacent organs, making surgical intervention difficult and increasing the risk of complications and recurrence (4-7).

Retroperitoneal tumors with histological diversity, excluding several lymphoid and reproductive system tumors, are not susceptive to radio- and chemotherapy $(8,9)$. A series of neoadjuvant therapies, pre- and/or intraoperation combined with radiochemotherapy, have been attempted to diminish the tumor volume of retroperitoneal tumors in previous phase I clinical trials; however, only limited clinical responses were observed $(8,10)$. Therefore, surgical excision remains the most effective treatment for retroperitoneal tumors. Complete removal is the goal of surgical intervention for retroperitoneal tumors $(4,5,7,11)$ and the type of incision should be carefully selected to ensure optimal exposure and sufficient surgical field visualization and to facilitate tissue dissection and separation (12).

In this study, we aimed to summarize, analyze and report 68 cases of patients with sizeable retroperitoneal tumors who underwent surgical treatment in our hospital between January, 1995 and June, 2013.

\section{Patients and methods}

General information. A total of 68 patients with sizeable retroperitoneal tumors, defined as tumors with boundaries beyond the midline of the abdomen (over the midline of the spine on imaging), were enrolled in the present study (Table I). A curved incision along the costal margin, or in combination with a longitudinal incision along the tumor boundary and the lower boundary of the tumor along the midline of the abdomen, were used for patients in group A (32 patients, 
male:female ratio $17: 15$, including 9 children), with a mean age of $56.7 \pm 12.8$ years (range, 22 months- 74 years). The longest diameter of the tumors ranged from 14 to $43 \mathrm{~cm}$, with a mean \pm standard deviation $(\mathrm{SD})$ of $21.3 \pm 4.4 \mathrm{~cm}$. Of the 32 patients, 17 had tumors on the left side and 15 on the right side. An abdominal incision or an oblique incision at the waist were used in group B, which included 36 patients (male:female ratio 19:17, including 11 children) with a mean age \pm SD of $54.2 \pm 12.1$ years (range, 20 months- 74 years). The longest diameter of the tumors ranged from 12 to $43 \mathrm{~cm}$, with a mean $\pm \mathrm{SD}$ of $22.3 \pm 3.4 \mathrm{~cm}$. Of these patients, 17 had tumors on the left side and 19 on the right side. A total of 14 patients (20.6\%) had benign and $52(69.4 \%)$ had malignant tumors. The clinical manifestations included abdominal pain in 22 cases, bloating in 34 cases, nausea, frequent or urgent urination and ipsilateral lumbar bulging in certain cases, whereas 12 patients were asymptomatic. The tumors in all the patients were located in the abdomen and across the midline of the abdomen. Preoperative computed tomography (CT)/magnetic resonance imaging (MRI) were used to measure the tumors and revealed that the tumors extended beyond the midline of the spine, exerting pressure on the gastrointestinal tract in 64 cases. Isotope renography was used to assess renal function in 60 cases, with 38 cases displaying renal and ureteral compression or obstruction and 6 cases of renal dysfunction. The function of the contralateral kidneys was normal. CT and MRI revealed abdominal aortic pressure and displacement in 21 cases, invasion and encapsulation in 11 cases, abdominal venous pressure and displacement in 32 cases, infiltration and encapsulation in 13 cases and encapsulation of the renal artery and vein in 15 cases. There were no significant differences between patients in groups A and B in terms of gender, tumor size, extent of infiltration around the left and right side, age and postoperative pathology. As regards preoperative preparation, $1,100 \mathrm{ml}$ blood was prepared on average per surgery and relevant consultations and coordination between departments were required to complete surgery in 15 cases.

Incision. For patients in group A, surgery was performed via a costal margin curved incision; a longitudinal incision was made along the tumor boundary and down to its lower boundary at the midline of the abdomen, as well as an incision at the rib outside the ipsilateral axillary line. When possible, the curve angle was not $<90$ degrees. For cases of superior renal and adrenal tumors, the prone position was used and the patients were elevated by 15 degrees ( 15 cases in total). For all other patients, the supine position was used. Following incision of the skin and abdominal fat layer, the muscle was separated at different levels to facilitate the identification of layers when suturing the incision, thus reducing the incidence of postoperative incisional hernia. The operative field was fully visualized and the anatomy and organizational associations were identified (1-10). In a region with relatively few blood vessels, a lateral peritoneal incision was performed outside the colon and the tumors were carefully separated. For retroperitoneal tumors, the surgical approaches may differ depending on the side of the tumor; the degree of complexity is higher in retroperitoneal tumors located on the right side, where important and easily-damaged organs, such as the liver, duodenum and vena cava, are located. In addition, the surgical field is more restricted. As a result, the most appropriate treatment was performed in conjunction with abdominal surgery with collaboration across multiple disciplines. For left-sided tumors, the pancreas, spleen with associated blood vessels and the aorta were identified and located prior to surgery. For tumors close to the mesenteric root, every effort was made to prevent damaging the superior mesenteric artery and vein.

For patients in group B, the incision was made at the midline of the abdomen or obliquely along the waist. The requirement for an additional incision for the purpose of obtaining the optimal surgical field was determined following abdominal exploration. If the tumor was too large and difficult to directly observe, the tumor and surrounding organs were assessed via the abnormal anatomical associations. The methods and techniques for isolating the tumors were the same as those used in group A patients.

Statistical analysis. A statistical analysis was performed using SPSS software, version 20.0 (IBM Corp., Somers, NY, USA) and the analysis of continuous variables between the two groups was performed using the Mann-Whitney U test. The comparison of categorical variables between the two groups was performed using the Chi-square test and the significance level was set at 0.05 .

\section{Results}

Tumor resection. Surgical resection was performed in the 32 patients in group A, of whom 22 underwent complete tumor resection, 1 underwent resection of the tumor capsule, 6 patients underwent sub-block resection, whereas complete resection with excision of adjacent organs was performed in 3 cases. In the cases undergoing complete resection, 1 patient required repair following injury of the vena cava and right renal vein and 1 patient required vena cava and aortic damage repair. Among the 36 cases in group B, surgical resection was performed in 25 patients, with complete resection achieved in 19 patients; resection of the tumor capsule was performed in 1 patient, sub-block resection was performed in 3 patients and removal of the tumor and surrounding organs was required in 2 patients. Surgical resection failed in 11 cases (6 patients were only subjected to pathological examination, 3 patients had $>50 \%$ residual tumor and 2 patients had $<50 \%$ residual tumor).

Transfusion and operative duration. The data regarding transfusion and operative time are presented in Table II. In group A, blood transfusion was performed in 19 cases, with a mean \pm SD of $400 \pm 200 \mathrm{ml}$ blood (range, 600-1,900 ml). The longest operative duration was $4.5 \mathrm{~h}$ and the shortest $2 \mathrm{~h}$, with a mean $\pm \mathrm{SD}$ of $3.5 \pm 0.5 \mathrm{~h}$. The weight of the resected tumor ranged between 3.5 and $10 \mathrm{~kg}$, with a mean $\pm \mathrm{SD}$ of $4.3 \pm 0.8 \mathrm{~kg}$. In group B, surgical tumor removal was performed in 30 patients (6 patients only received pathological examination) and 25 patients were transfused with a mean \pm SD of $1,100 \pm 200 \mathrm{ml}$ blood (range, 400-4,500 ml). The mean $\pm \mathrm{SD}$ operative duration was $5.8 \pm 0.7 \mathrm{~h}$ (range, 10-3.5 h) and the weight of the resected tumor varied from 1.2 to $7.9 \mathrm{~kg}$, with a mean \pm SD weight of $2.4 \pm 0.7 \mathrm{~kg}$. 
Table I. Pathological considerations.

\begin{tabular}{lccc}
\hline Tumor types & $\begin{array}{c}\text { Cases } \\
(\mathrm{n}=68)\end{array}$ & $\begin{array}{c}\text { Maximal diameter, } \\
\text { mean }(\mathrm{cm})\end{array}$ & $\begin{array}{c}\text { Left } \\
(\mathrm{n}=35)\end{array}$ \\
\hline Adrenal cortical carcinoma & 5 & 14 & 2 \\
$(\mathrm{n}=33)$
\end{tabular}

Table II. General and surgery-related information from the two groups of patients.

\begin{tabular}{lccc} 
Items & $\begin{array}{c}\text { Group A (costal margin } \\
\text { curved incision) }\end{array}$ & $\begin{array}{c}\text { Group B (mid-abdominal, } \\
\text { waist oblique incision) }\end{array}$ & P-value \\
\hline Age (years) & $56.7 \pm 12.8$ & $54.2 \pm 12.1$ & 0.274 \\
Location, left/right (no. of cases) & $17 / 15$ & $19 / 17$ & $0.140 / 0.138$ \\
Maximum tumor diameter (cm) & $21.3 \pm 4.4$ & $22.3 \pm 3.4$ & 0.224 \\
Operative time (h) & $3.5 \pm 0.5$ & $5.8 \pm 0.7$ & 0.023 \\
Transfused blood volume (ml) & $400 \pm 200$ & $1100 \pm 200$ & $<0.001$ \\
Post-surgical hospitalization (days) & 8.5 & 11.5 & $<0.001$ \\
Surgically resected tumor weight (kg) & $4.3 \pm 0.8$ & $2.4 \pm 0.7$ & 0.012 \\
Perioperative deaths (no. of cases) & 0 & 1 & 0.034 \\
Intestinal fistula after surgery (no. of cases) & 0 & 2 & 69.4 \\
Resection rate (\%) & 100 & &
\end{tabular}

Values are presented as mean \pm standard deviation.

Perioperative complications. There were no reported deaths or development of intestinal fistulas in group A. The patients were discharged from the hospital after a mean of 8.5 days after surgery. In group B, 1 patient succumbed to pneumonia and intestinal fistulas developed in 2 patients. The patients were discharged from the hospital after a mean of 11.5 days after surgery (Table II).

Statistical analysis. Variables such as patient age, location of the tumor and tumor size were not statistically significantly different between groups $\mathrm{A}$ and $\mathrm{B}(\mathrm{P}=0.274,0.138$ and 0.224 , respectively; Table II), indicating that the basic characteristics of the patients were comparable between the two groups. However, there were statistically significant differences regarding operative time, volume of transfused blood, postoperative in-hospital stay, weight of surgically removed tumor, perioperative mortality, development of intestinal fistula after surgery and surgical resection rate in any pairwise comparison (Table II), indicating that a curved incision under the costal margin was superior to the abdominal midline or oblique waist incision, in terms of surgical resection rate and safety.

\section{Discussion}

Retroperitoneal surgery remains the only viable curative approach for the treatment of retroperitoneal tumors $(3,5,9)$. When retroperitoneal tumors become sizeable, the digestive system and respiratory function may be affected, due to displacement of the surrounding organs and anatomical pressure, which may also lead to severe obstruction and dysfunction of the urinary system. Moreover, the majority of retroperitoneal tumors are malignant and not sensitive to radiotherapy or chemotherapy. Therefore, surgery is often the only way to yield any therapeutic effect $(7,10,13)$. Preoperative tests, particularly CT/MRI, allow for determination of tumor location, size and its association with the surrounding organs and blood vessels. Other preoperative examinations include tests of renal, blood clotting and respiratory function, as well as other routine examinations $(1,2,5,7)$. A good preoperative consultation and multidisciplinary collaboration constitute the basis for an uneventful surgery. Due to the extensive area involved in retroperitoneal tumor surgery, the risks of hemorrhage and vascular injury, as well as sudden decompression of blood vessels following resection of a sizeable tumor, may 
result in hypovolemic shock; therefore, adequate volumes of blood must be prepared $(3,5,13)$. The majority of available reports emphasize the significance of adequately exposing the operative field. Due to the association of retroperitoneal tumors with surrounding tissues, there is often severe compression and even infiltration or encapsulations of non-adjacent organs, thus increasing the difficulty of identifying tissues and anatomical associations during surgery $(1-7,11,13,14)$. Retroperitoneal tumors located on the left or the right side of the body require different approaches. The degree of complexity of surgeries for sizeable retroperitoneal tumors on the right side is generally higher, due to the presence of important and easily-damaged organs, such as the liver, duodenum and vena cava. During tumor removal on the left side, the pancreas, spleen and associated blood vessels and the aorta must be clearly identified. Tumors close to the mesenteric root must also be treated with extra caution to prevent superior mesenteric artery and vein damage. Starting with an area containing less vasculature, the peritoneal incision is performed while trying to identify the separating interfaces and potential gaps between the tumor and its surrounding tissues. Engorged vascular tumor surfaces must also be avoided. For sizeable retroperitoneal tumors, the size of the tumor determines the anatomical area involved in surgical separation. In areas with less vasculature, for example near the dorsolateral aspect of the tumor or at the anterolateral musculature, the incision may be performed quickly near the tumor surface to reduce surgical separation time. Blood vessels that cannot be avoided require hemostasis. For areas that are difficult to reach, gauze may be applied with pressure to stop bleeding, particularly of venous origin. The arteries around the tumor must be identified to reduce arterial bleeding. For possible vascular injuries, good preparation and timely treatment are crucial, including pre-blocking, ligation, repair and reconstruction $(3-7,11)$. Whether the removal of sizeable retroperitoneal tumors is required may be determined following conventional exploration rather than following laparotomy. At the time of the surgery, the surgeon must be able to clearly identify the extent of the association between the tumor and its surrounding organs to assess whether removal is required. For planned surgical resection of surrounding organs, the surgeon must constantly analyze and observe the anatomical shift to determine whether the planned organ resection should be performed and have timely discussions with relevant departmental colleagues. Ensuring a good surgical field is the basis for the success of the operation and the aim of this study was to select the type of incision that ensures the optimal surgical field.

By analyzing these results, we have determined that a curved incision under the costal margin is superior to an oblique incision along the waist in terms of surgical resection rate, blood transfusion volume, operative time, hospital stay following surgery, perioperative mortality, surgical quality and safety; however, the extent of its long-term effects on retroperitoneal tumor treatment requires further observation in the future.

\section{References}

1. He YL: Retroperitoneal tumor treatment design and preoperative preparation. Chin J Pract Surg 28: 256-258, 2008 (In Chinese).

2. Tian W, Song S, Liang F, Chen L, Jiang Y and Fu W: Reoperative treatment of recurrent retroperitoneal tumor. Chin J Surg 36: 221-223, 1998 (In Chinese).

3. Xu L, Wan J and Wang X: Analysis of 37 cases urinary systemrelated retroperitoneal tumor. Chin J Prim Med Pharm 11: 864-865, 2004 (In Chinese).

4. Dai W and Yu HZ: Surgical treatment of huge retroperitoneal tumor: A report of 22 cases. Anhui Med J 31: 1197-1198, 2010 (In Chinese).

5. Liang FQ: Retroperitoneal tumor resection and vascular occlusion and reconstruction during surgery. Chin J Pract Surg 28: 301-303, 2008 (In Chinese).

6. Qu XL and Chen FZ: Resection of retroperitoneal tumors with reconstruction of major blood vessels in 12 patients. Chin Oncol 18: 234-236, 2008 (In Chinese).

7. Wan YL and Tang J: The prevention and treatment of bleeding during retroperitoneal tumor surgery. Chin J Pract Surg 28: 286-287, 2008 (In Chinese).

8. Chen L and Wei B: Primary retroperitoneal tumor combined multivisceral resection. Chin J Pract Surg 28: 263-265, 2008 (In Chinese).

9. Rao XF, Cao H, Liao CW, et al: Diagnosis and treatment of Primary retroperitoneal liposarcoma. Chin J Gen Surg 19: 1166-1169, 2010 (In Chinese).

10. Pisters PW and O'Sullivan B: Retroperitoneal sarcomas: Combined modality treatment approaches. Curr Opin Oncol 14: 400-405, 2002.

11. Huang $\mathrm{H}, \mathrm{Wu}$, Hong $\mathrm{S}$, et al: The anatomic location of retroperitoneal tumors and their clinical diagnosis. J Pract Med 24: 1376-1378, 2008

12. Chen L and Bian SB: Attention to surgical management strategies of complex retroperitoneal tumors. Chin J Pract Surg 33: 811-813, 2013 (In Chinese).

13. Hao YJ and Luo CH: The current situation and progress of the diagnosis and treatment of retroperitoneal liposarcoma. J Surg Concepts Pract 17: 398-401, 2012 (In Chinese).

14. Wang J: Retroperitoneal tumor pathological type, histological feature and prognosis. Chin J Pract Surg 28: 249-252, 2008 (In Chinese). 